\title{
Structure of the SMC
}

\section{Stellar component distribution from 2MASS data}

\author{
I. Gonidakis ${ }^{1}$, E. Livanou ${ }^{1}$, E. Kontizas ${ }^{2}$, U. Klein ${ }^{3}$, M. Kontizas ${ }^{1}$, M. Belcheva ${ }^{1}$, P. Tsalmantza ${ }^{4}$, and A. Karampelas ${ }^{1}$
}

1 Department of Astrophysics Astronomy \& Mechanics, Faculty of Physics, University of Athens, 15783 Athens, Greece e-mail: elivanou@phys.uoa.gr

2 Institute for Astronomy and Astrophysics, National Observatory of Athens, PO Box 20048, 11810 Athens, Greece

3 Radioastronomisches Institut der Universitat Bonn, Auf dem Hogel 71, 53121 Bonn, Germany

4 Max-Planck-Institut fur Astronomie, Konigstuhl 17, 69117 Heidelberg, Germany

Received 21 March 2008 / Accepted 24 November 2008

ABSTRACT

\begin{abstract}
Aims. The spatial distribution of the SMC stellar component is investigated from 2MASS data. The morphology of the different age populations is presented. The center of the distribution is calculated and compared with previous estimations. The rotation of the stellar content and possible consequence of the presence of dark matter is discussed.

Methods. The different stellar populations are identified through a CMD diagram of the 2MASS data. Isopleth contour maps are produced in each case, to reveal the spatial distribution. The derived density profiles are discussed.

Results. The older stellar population follows an exponential profile at projected diameters of about $5 \mathrm{kpc}\left(\sim 5^{\circ}\right)$ for the major axis and $\sim 4 \mathrm{kpc}$ for the minor axis, centred at RA: $0^{\mathrm{h}} 51^{\mathrm{min}}$, Dec: $-73^{\circ} 7^{\prime}(\mathrm{J} 2000.0)$. The centre coordinates are found to be the same for all the different age population maps and are in good accordance with the kinematical centre of the SMC. However they are found to be considerably different to the coordinates of the centre of the gas distribution. The fact that the older population is found in an exponential disk suggests that the stellar content is rotating, a possible consequence of dark matter presence. The strong interactions between the MCs and the MilkyWay might explain the difference in the distributions of the stellar and gas components. The lack of an observed velocity element, which implies an absence of rotation and contradicts the consequences of an exponential profile of the stellar component, may also be a result of gravitational interactions.
\end{abstract}

Key words. galaxies: Magellanic Clouds - galaxies: structure - cosmology: dark matter

\section{Introduction}

The Small Magellanic Cloud (SMC) is classified as an irregular dwarf galaxy, characterized by a pronounced central feature of the Bar and an eastern extension called the Wing. There has been a lot of investigation carried out studying the distribution of different stellar populations of this galaxy. Initially, Freeman (1970) calculated the scale length of the exponential component of the SMC from $B$-magnitude photometry. He found $a^{-1}=0.63 \mathrm{kpc}$. Later, Gardiner \& Hawkins (1991) found an exponential profile in a northern direction with $a^{-1}=1.2 \mathrm{kpc}$, describing well the distribution up to $r=6 \mathrm{kpc}$. However this value was calculated over the observed projected radial distribution assuming a spherically symmetric halo structure.

Morgan \& Hatzidimitriou (1995) have carried out a spectroscopic survey of carbon stars in the outer parts of the SMC. Regarding the spatial distribution of these stars, they conclude that at most surface densities the SMC appears elliptical with the major axis parallel to the Bar, but there is a significant northward distortion of the outer contours. The diameter of the outermost contour is $\sim 10^{\circ}-12^{\circ}$. The authors also underline the existence of a spiral-arm-like extension of the carbon star distribution southwards at (projected) distances greater than $4^{\circ}(\sim 4 \mathrm{kpc})$ from the optical centre, although its origin is not clear.

Counts of sources towards the Magellanic Clouds from the DENIS near-infrared survey have been studied by Cioni et al. (2000). Their investigation was based on differentiation in the $(I-J, I)$ colour magnitude diagram (CMD) to distinguish between three groups of objects with different mean ages. The spatial distribution of the three age groups is quite different: the youngest stars exhibit an irregular distribution while the older stars are smoothly and regularly distributed. The AGB and RGB stars are characterised by a regular but double peaked structure, an offset from the HI distribution, and a mean age difference of the two maxima of their star count map.

Maragoudaki et al. (2001) studied the spatial distribution of the SMC stellar population according to their age, based on optical data. They have shown that the older stellar population shows a rather regular and smooth distribution, while the youngest stellar component (age less than $8 \times 10^{6} \mathrm{yr}$ ) appears mainly along the northeast-southwest direction, forming the Bar.

Stanimirovic et al. (2004) in a detailed study of the HI kinematics conclude that the HI velocity field shows a large velocity gradient from the south west to the north east. The isovelocity contours of this velocity field show some symmetry, suggestive of a differential rotation. Some large-scale distortions in this velocity field are easily visible but could be related to positions of several supergiant shells. In the same article the authors conclude that, in contrast to the HI distribution, the old stellar populations appear to have a spheroidal spatial distribution and a total absence of rotation.

In order to further investigate this discrepancy between the gas and stellar component distributions, we have studied the distribution of the various stellar components of the SMC from near IR surveys. We know that IR observations characterize the mass 
of the galaxy better than those in shorter wavelengths where absorption masks a large part of the stellar component. A detailed study of the stellar component as it is revealed from 2MASS is given in Sect. 2. A comparison with available radio data is given in Sect. 3, implementing the same approach used for the near IR data. Discussion and conclusions are given in Sect. 4.

\section{2MASS data}

The near-infrared (NIR) images were obtained by the twomicron all-sky survey (2MASS), an ongoing effort to map the entire sky at $J$-band ( 1.25 microns), $H$-band ( 1.65 microns) and $K$ s-band (2.17 microns) wavelengths. The 2MASS survey is led by the University of Massachusetts, with all data and images processed at Caltech's infrared processing and analysis centre (IPAC). The survey utilizes two nearly identical $1.3-\mathrm{m}$ telescopes located at Mount Hopkins (Arizona) and at Cerro Tololo (Chile). While the pixel size is 2.0 arcsec, the survey strategy of over-sampling yields an effective resolution of about 1 arcsec. Exposure times are $7.8 \mathrm{~s}$. 2MASS has uniformly scanned the entire sky in three near-infrared bands to detect and characterize point sources brighter than about $1 \mathrm{mJy}$ in each band, with a signal-to-noise ratio (SNR) greater than 10. This has achieved an 80000 -fold improvement in sensitivity relative to earlier surveys. The data used here are from the 2MASS All-Sky point source catalog (PSC) (fp_psc), at the IPAC infrared science archive (IRSA), Caltech/JPL (http://irsa.ipac.caltech. edu/applications/Gator).

IRSA gives choices for the origin of the default magnitudes and uncertainties in each band. We have used only sources with a satisfying quality of photometry in the $J, H$ and $K$ s bands. For this, we choose the case where the default magnitude is derived from a profile-fitting measurement made on the $1.3 \mathrm{~s}$ "Read_2" exposures. The profile-fit magnitudes are normalized to curveof-growth-corrected aperture magnitudes. This is the most common type in the PSC, and is used for sources that have no saturated pixels in any of the $1.3 \mathrm{~s}$ exposures.

The examined area of the SMC is $6.9^{\circ} \times 12^{\circ}$. Data from a small near-by field of $2.1^{\circ} \times 1^{\circ}$ are also obtained to use later in calculating the Galactic contribution. The coordinates of this near-by field are $47.5 \leq \mathrm{RA} \leq 60$ and $-68 \leq$ Dec $\leq-67$. It was selected to be close, but out of the SMC field, in order to represent well the distribution of background stars near the SMC. The dimensions were chosen to be large enough for adequate statistics for the SMC field. The initial catalogues contain 359234 stars for the SMC area and 10444 stars for the small near-by field. The total photometric uncertainty for 2MASS data is given by IRSA (details at http://www.ipac.caltech.edu/2mass/releases/ allsky/doc/sec4_5e.html). Sources with a total photometric uncertainty greater than or equal to $0.2 \mathrm{mag}$ for any of the $J, H$ and $K$ s bands were excluded, providing more reliable photometric measurements. The catalogues used contained 293330 stars for the SMC field and 8428 stars for the small nearby field.

\subsection{Identifying stellar populations}

Major stellar populations are identified based on matching features of the observed colour-magnitude diagram with expected positions of known populations. Based on Hiparchos data of SMC stars, Gavras (2003) has determined the criteria for the different star types on the $K$ s versus $J-K$ s CMD of the SMC. The A, B type stars are found at $-1<J-K \mathrm{~s} \leq 0.2$ and $K \mathrm{~s} \leq 15$. The

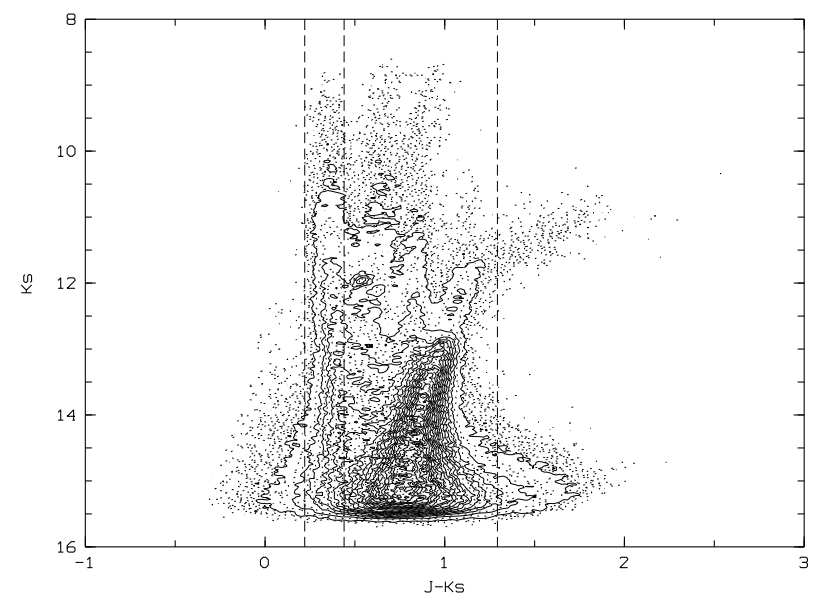

Fig. 1. Colour Magnitude Diagram $K$ s vs. $J-K$ s, of the SMC, from 2MASS.

F and $\mathrm{G}$ types have $0.2<J-K \mathrm{~s} \leq 0.45$ and $K \mathrm{~s} \leq 15$. The $\mathrm{K}, \mathrm{M}$ and faint carbon stars have $0.45 \leq J-K \mathrm{~s} \leq 1.3$ and $K \mathrm{~s} \leq 15$ and carbon stars have $J-K \mathrm{~s} \geq 1.3$ and $K \mathrm{~s} \leq 15$. Applying the criteria above to the 293330 stars for the SMC field we find $2618 \mathrm{~A}$, B type stars, $30042 \mathrm{~F}$ and $\mathrm{G}$ type, $177471 \mathrm{~K}, \mathrm{M}$ and faint carbon stars and 4705 carbon stars.

Two CMDs, the $K$ s vs. $J-K$ s for the SMC area and the nearby field, were produced. Both diagrams are divided in a grid with the same number of cells $(22 \times 16)$. The cells have dimensions that provide a fine grid, while they include enough stars for adequate statistics. The grids have the same number of cells and same starting and ending points for each axis, in order to compare numbers of stars with the same $K$ s and $J-K$ s values. The cells of the near-by field were normalized to the equal area of the cells of the SMC area and their stars considered as background stars were subtracted randomly. The CMD of $K$ s versus $J-K$ s for the SMC field after subtraction of the background contribution is shown in Fig. 1. The criteria set by Gavras (2003) for $J-K$ s values are indicated by the dashed lines.

Since the different populations were defined, star counts were performed in a grid of $288 \times 500$ pixels for the SMC region. The isopleth contour maps of the SMC field that were produced for each of the four cases can be seen in Fig. 2. It is very interesting how well the old population (bottom right in Fig. 2) reveals the center of mass of this stellar population.

\subsection{Distribution of late type stars}

In the case of $\mathrm{K}, \mathrm{M}$ and faint carbon stars the distribution reveals the elliptical structure of the disk. The number density of the stars versus the distance from the centre has been estimated. The isopleth contour map of the SMC field was reproduced (Fig. 3) with larger steps between the contours in order to allow a more accurate selection of the image points that will be considered. The outermost contour is the same as in Fig. 2 and will be the one specifying the diameter of each structure. We selected 4 directions a, b, c and d along the "minor" and "major" axis to derive their density profiles. The "major" axis is the one following the direction of the Bar, from north-east to south-west, while the "minor" axis is from south-east to north-west.

Fitting exponential curves to the diagrams of the number density of sources $(N)$ versus (projected) distance from the centre of the galaxy $(r)$, with exponential functions was very 

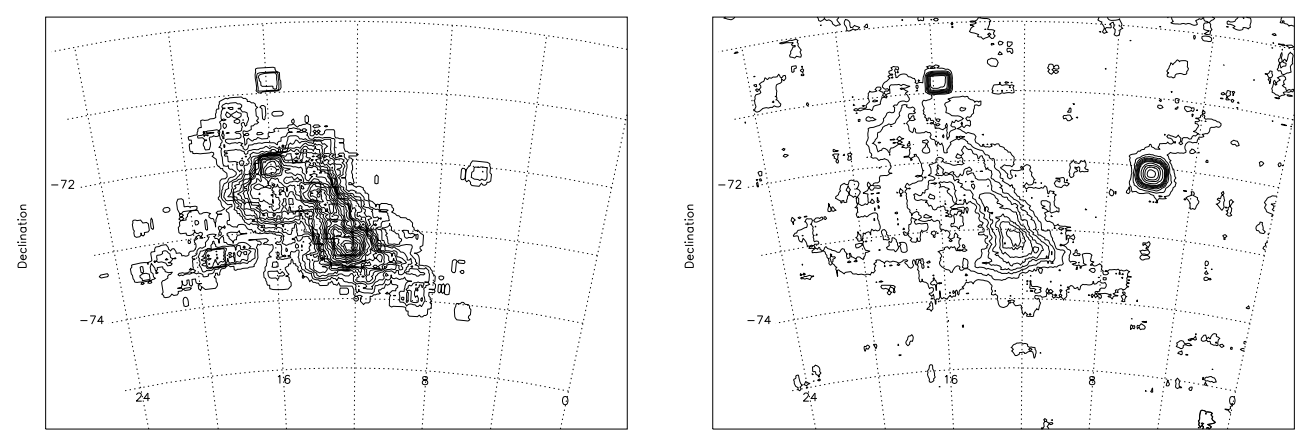

Right Ascension
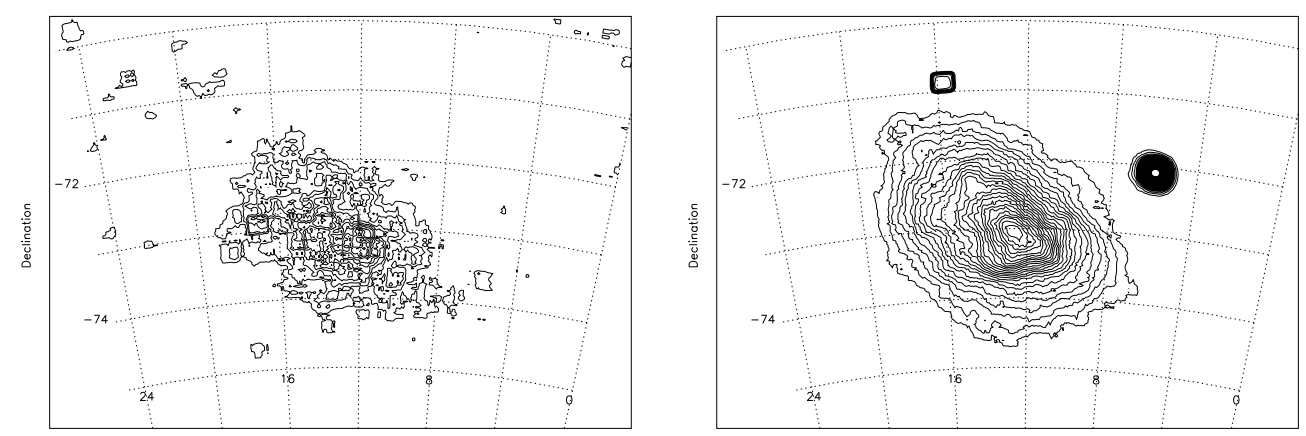

Right Ascension

Right Ascension

Fig. 2. Isopleth contour maps of the SMC different stellar populations, from 2MASS. Top left: A and B type stars, top right: F and G, bottom left: Carbon stars and bottom right: $\mathrm{K}, \mathrm{M}$ and faint carbon stars.

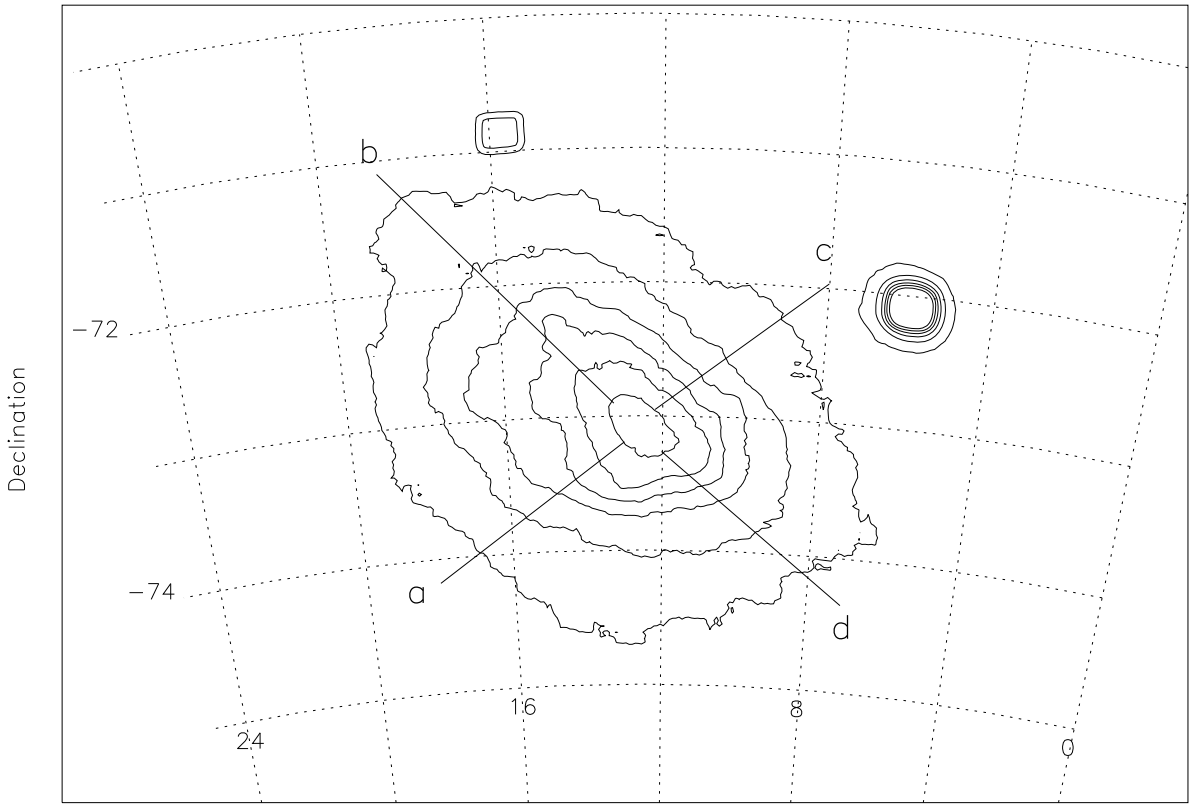

Right Ascension
Fig. 3. Isopleth contour map of the SMC K, M and faint carbon stars, from 2MASS. Lines indicate the selected directions. The "major" axis of the galaxy is the one following the direction of the Bar, from north-east to south-west, while the "minor" axis is from south-east to northwest. satisfying. The best fit functions are $Y=15.9 \pm 2.2 \exp (-1.0 \pm$ $0.5 X)-0.8 \pm 3.1, Y=19.7 \pm 6.8 \exp (-0.4 \pm 0.3 X)-4.7 \pm 8.1$, $Y=16.6 \pm 1.8 \exp (-1.5 \pm 0.6 X)+0.3 \pm 2.0, Y=17.8 \pm$ $2.0 \exp (-1.3 \pm 0.5 X)-0.1 \pm 2.1$, for the directions $\mathrm{a}, \mathrm{b}, \mathrm{c}$ and $\mathrm{d}$ respectively. The values of $a^{-1}$ are 1.0, 2.5, 0.7 and 0.8 derived for each direction respectively, corresponding to a mean value of $a^{-1}=1.25 \mathrm{kpc}$. For the power-law scenario, the best fit functions are $Y=5.0 \pm 0.5 X^{(-0.6 \pm 0.1)}, Y=6.8 \pm 0.5 X^{(-0.6 \pm 0.1)}$,
$Y=4.2 \pm 0.6 X^{(-0.8 \pm 0.1)}, Y=5.0 \pm 0.5 X^{(-0.8 \pm 0.1)}$, for the directions $\mathrm{a}, \mathrm{b}, \mathrm{c}$ and $\mathrm{d}$ respectively. The chi-squared parameters are $0.04,0.03,0.09$ and 0.11 in the case of exponential fitting curves for the directions a, b, c and d respectively. The corresponding values for the power-law case are 1.6, 2.4, 1.0 and 2.4, indicating that exponential fitting better represents the data. Consequently the mass distribution derived here is described by an exponential disk as illustrated in Fig. 4 with the best fitting curves. Thus, 

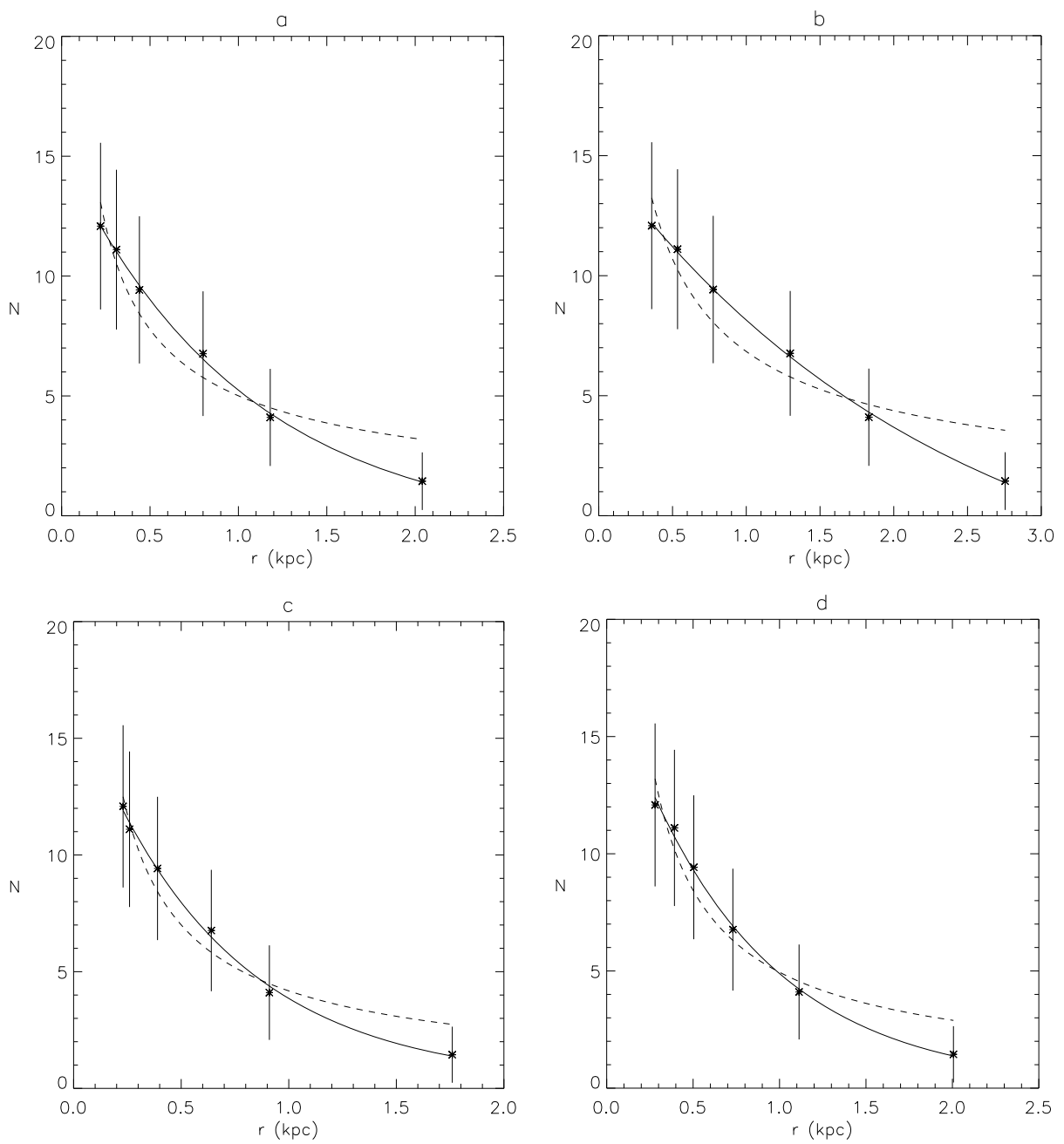

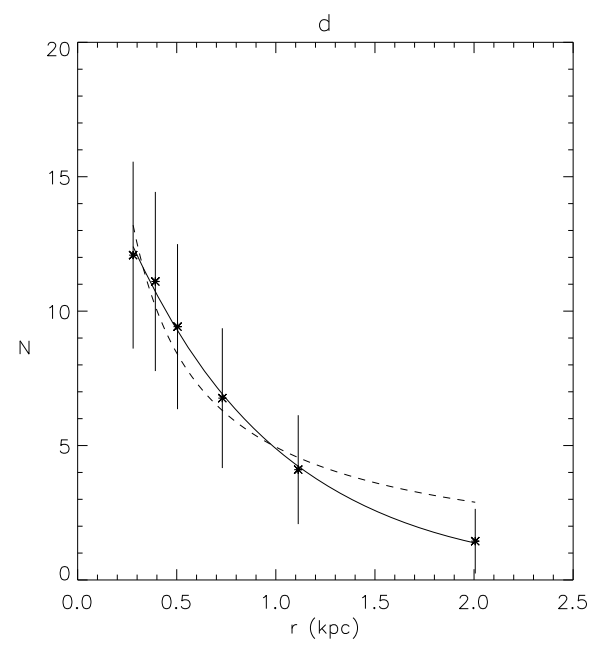

Fig. 4. Number of stars per pixel $(N)$ vs. distance from the centre $(r)$ (star symbols), fitted with exponential curves (solid lines) and power-law curves (dashed lines), for 2MASS data for the $\mathrm{a}, \mathrm{b}, \mathrm{c}$ and d directions respectively.
2MASS reveals an exponential profile for the older stellar population with a (projected) diameter $\sim 3.8 \mathrm{kpc}$ for the "minor" axis and $\sim 4.8 \mathrm{kpc}$ for the "major" axis. The center of the elliptical distribution is best determined by the right lower map of Fig. 2, at RA: $0^{\mathrm{h}} 51^{\mathrm{m}}$, Dec: $-73^{\circ} 7^{\prime \prime} .2$, that represents the K, M and faint carbon stars.

The exponential disk model for $\mathrm{K}, \mathrm{M}$ and faint carbon stars, has to face the fact that the values of $a^{-1}$ are not the same for the semi-axis, and the fact that the exponential along direction $(d)$ is measured to be "steeper" than the exponential in direction (a). The observed asymmetry might be explained by the inclination of the SMC, although the deprojection is not clear enough in the case of this galaxy. However further investigation should shed light on this issue.

\section{Radio data}

A radio image of the $\mathrm{SMC}$ at $1400 \mathrm{GHz}(21 \mathrm{~cm})$ was obtained from Uli Klein (private communication) based on the data that were derived from the work by Haynes et al. (1986). The data represent well the extended radio envelope of the SMC, well beyond any site of obvious active star formation and are appropriate for investigating the gas distribution in the SMC. The radial profile of this image is presented in Fig. 5. Using the previous methodology we created diagrams of flux density $(F)$ versus distance from the centre $(r)$, and fitted data with exponential and power-law curves in order to reveal the most efficient mathematical description of this distribution. The best fit exponential functions we found are $Y=1208.1 \pm 2.0 \exp (-2.2 \pm 0.01 X)+$ $30.7 \pm 1.9, Y=17243.6 \pm 270.6 \exp (-3.7 \pm 0.02 X)+28.2 \pm 2.1$, $Y=1801.9 \pm 21.2 \exp (-1.33 \pm 0.02 X)-585.6 \pm 14.6$, and $Y=248641.0 \pm 6690.8 \exp (-4.5 \pm 0.02 X)+60.13 \pm 1.6$, for the directions $\mathrm{a}, \mathrm{b}, \mathrm{c}$ and $\mathrm{d}$ respectively. The values of $a^{-1}$ are $0.45,0.27,0.75$ and 0.22 derived for each direction, corresponding to a mean value of $a^{-1}=0.42 \mathrm{kpc}$. For the power-law case, the best fit functions are $Y=241.6 \pm 0.5 X^{(-0.8 \pm 0.001)}$, $Y=456.9 \pm 0.5 X^{(-3.4 \pm 0.01)}, Y=191.7 \pm 0.5 X^{(-0.9 \pm 0.002)}$, $Y=3087.4 \pm 10.8 X^{(-5.5 \pm 0.01)}$, for the directions a, b, c and d. The chi-squared parameters are 590.0, 390.8, 33.8 and 471.9 in the case of exponential fitting curves for the directions a, b, c and d, whereas the corresponding values for the power-law case are 5542.13, 11285.9, 188.0 and 419.5. The comparison of the two sets of parameters allows us to suggest that exponential fitting better represents the distribution. We notice, however, that in for direction (d) a power-law is more efficient. Consequently, the mass distribution derived here is described by an exponential disk, as illustrated in Fig. 6, with the best fitting curves.

This led us to a mean value of $a^{-1}=0.42 \mathrm{kpc}$ for the radio data. The centre is found at RA: $0^{\mathrm{h}} 55^{\mathrm{m}} 7^{\mathrm{s}} .2$, Dec: $-72^{\circ} 47^{\prime} 59^{\prime \prime}$. The HI exponential profile has a scale length of $0.45 \mathrm{kpc}$ along the south-east direction (a), $0.27 \mathrm{kpc}$ to the north-east direction (b), $0.75 \mathrm{kpc}$ to the north-west (c) and $0.22 \mathrm{kpc}$ to the southwest (d). These coordinates of the center are in accordance with 

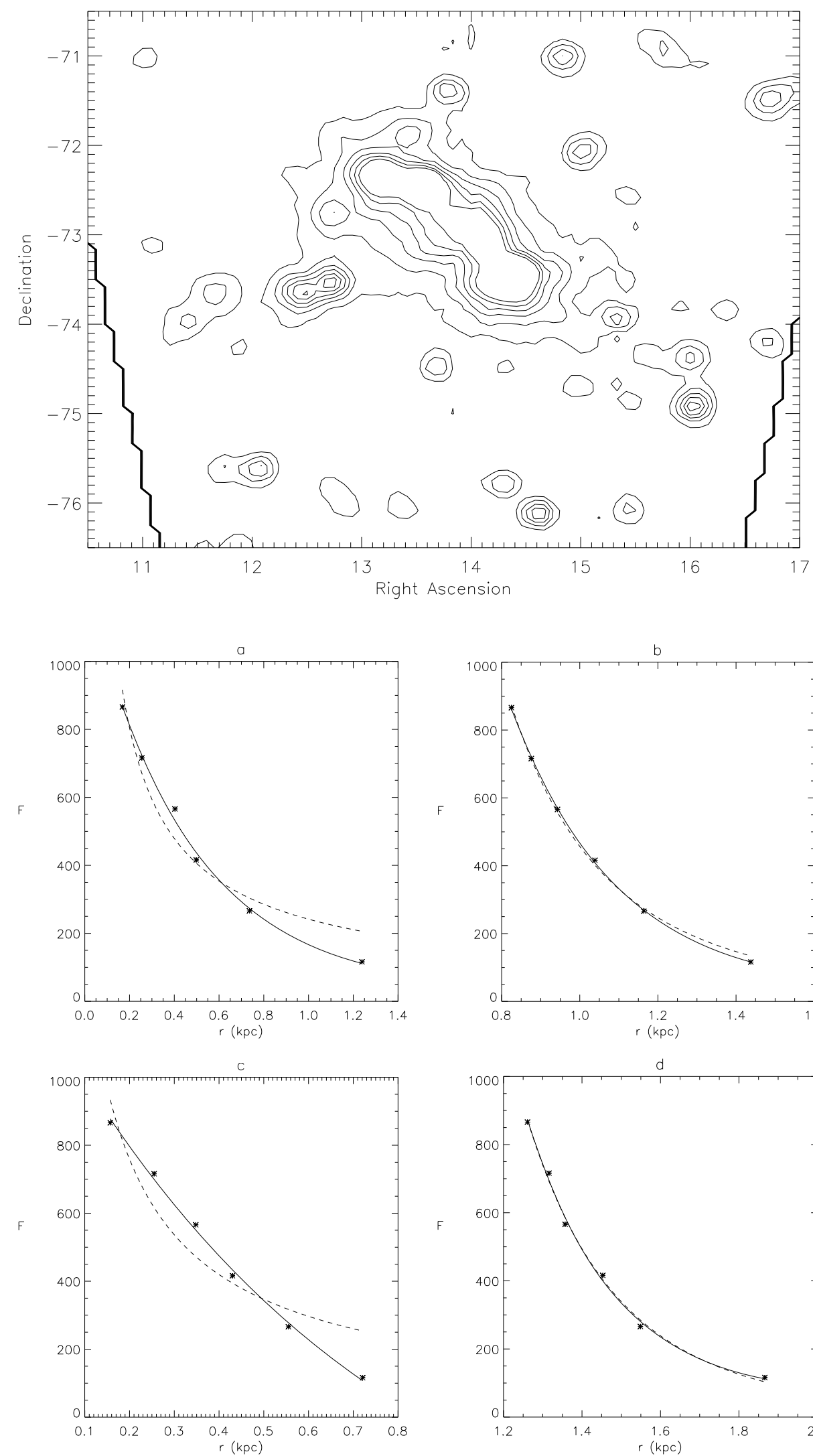
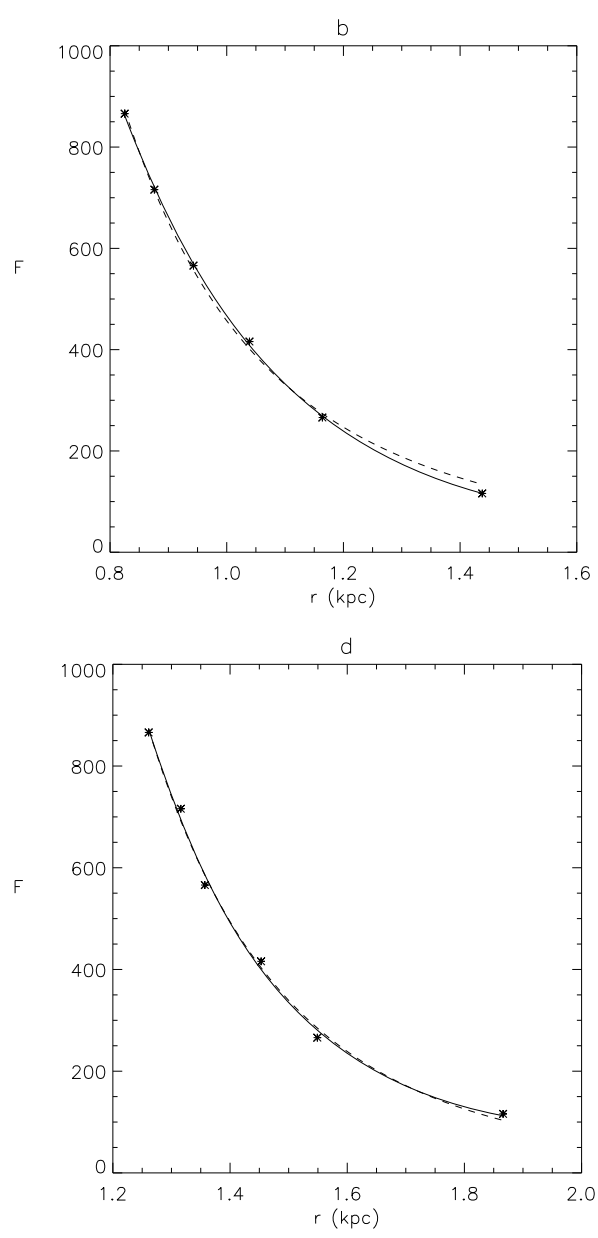

Fig. 5. Isodensity contour map of the SMC radio data.

Fig. 6. Flux density $(F)$ versus distance from the centre $(r)$ (star symbols), fitted with exponential curves (solid lines) and power-law curves (dashed lines), for radio data for the a, $\mathrm{b}, \mathrm{c}$ and d directions.

the results of Stanimirovic et al. (2004) derived from higher resolution data, and confirms that the differences found here between the central coordinates of the stellar and the gas component are real.

\section{Discussion and conclusions}

The distribution of the stellar components in the near IR (2MASS) has been used to investigate the radial distribution of 
the stellar mass in the SMC. The isopleths in near IR wavelengths are expected to represent more accurately the distribution of stars since the observations are less affected by absorption.

In the case of the 2MASS data we set the criteria for collecting the different stellar populations and present their spatial distributions. It has been found that an exponential profile better fits the distribution of the older population of the SMC. In Fig. 2, the bottom right map of K, M and faint carbon stars reveals extremely accurately the center of the mass distribution of the oldest stellar population of the SMC; the coordinates of this center are found to be the same for all cases, independently of the age of the population they represent.

This centre of the isopleths is found to be RA: $0^{\mathrm{h}} 51^{\mathrm{min}}$, Dec: $-73^{\circ} 7^{\prime}$ and the mean value for the scale length is $a^{-1}=1.25 \mathrm{kpc}$.

\subsection{The center offset}

An important characteristic of the distribution of the older stellar population of the SMC is the offset of the center of its distribution from the centre of the distribution found from HI data (Stanimirovic et al. 2004). In a more recent work, Piatek et al. (2007) calculated the kinematical centre of the SMC at RA: $0^{\mathrm{h}} 52^{\mathrm{m}} .8$, Dec: $-72^{\circ} 30^{\prime}$ from HST data, in good agreement with our calculations. They also explain that the presence of the Bar or a strong tidal disturbance confer extra streaming motion to a system and thus old and young populations can have distinct kinematics. The strong interactions of the SMC with the LMC and the MilkyWay that have probably contributed to the formation of the Bar (Maragoudaki et al. 2001) could also have as a result the observed difference in the centres of the distributions of the stellar and the gas component as well as the small radial velocity gradient among SMC stars (Bekki \& Chiba 2008), that contradicts the exponential profile of the stellar component.

Stanimirovic et al. (2004) suggest that according to SMC rotation curve, a dark matter halo is not needed to explain itt's dynamics. However, reconsidering the values of the center of the SMC and adopting the center of the mass derived above when calculating rotational velocity profiles may be important considering the issue of dark matter presence in this galaxy. SMC is a dwarf irregular galaxy, which are expected to be dark matter dominated (Cote et al. 2000; Begum et al. 2006; Strigari et al. 2008). However, rotational velocities do not support this case for the SMC, probably because they have been affected by the interactions of the Magellanic system with the Milky way.

\subsection{The scale length values}

Freeman (1970) investigated the structure of the SMC through bright stars distribution and found that their elliptical component is characterised by a scale length of $a^{-1}=0.63 \mathrm{kpc}$. We find an exponential profile for the older stellar population with $a^{-1}=$ $1.25 \mathrm{kpc}$, while the gas exponential disk has $a^{-1}=0.42 \mathrm{kpc}$. These three values well represent the structure of three different galactic components and are in accordance with the findings that gas (radio data) is more concentrated in a thinner disk, younger stars (best represented by $B$-magnitude distribution) lie on a thicker disk and the oldest stars reach greater distances from the center.

Acknowledgements. The authors would like to thank the University of Athens (ELKE) for partial financial support. The project is co-funded by the European Social Fund and National Resources-(EPEAEK II) PYTHAGORAS II.

\section{References}

Begum, A., Chengalur, J. N., Karachentsev, I. D., Kaisin, S. S., \& Sharina, M. E. 2006, MNRAS, 365, 1220

Bekki, K., \& Chiba, M. 2008 [arXiv:0806.4657]

Cioni, M.-R. L., Habing, H. J., \& Israel, F. P. 2000, A\&A, 358, L9

Cote, S., Carignan, C., \& Freeman, K. C. 2000, AJ, 120, 3027

Freeman, K. C. 1970, ApJ, 160, 811

Gardiner, L. T., \& Hawkins, M. R. S. 1991, MNRAS, 251, 174

Gavras, P. 2003, MSc thesis, University of Athens

Haynes, R. F., Klein, U., Wielebinski, R., \& Murray, J. D. 1986, A\&A, 159, 22

Maragoudaki, F., Kontizas, M., Morgan, D. H., et al. 2001, A\&A, 379, 864

Morgan, D. H., \& Hatzidimitriou, D. 1995, A\&AS, 113, 539

Piatek, S., Pryor, C., \& Olszewski, E. W. 2007 [arXiv: 0712 . 1764]

Stanimirovic, S., Staveley-Smith, L., \& Jones, P. A. 2004, ApJ, 604, 176

Strigari, L. E., Koushiappas, S. M., Bullock, J. S., et al. 2008, ApJ, 678, 614 